\title{
Wege zur nachhaltigen Ökonomie
}

\author{
Während die Bedeutung eines wirkungsvollen Umweltschutzes heute für eine \\ übergroße Mehrheit der deutschen Bevölkerung und die Mehrzahl der Wissen- \\ schaftler mit Ausnahme der Ökonomen einen Konsens darstellt, wird über die \\ adäquaten Strategien hierfür immer noch heftig debattiert. Der Artikel geht der \\ Frage nach, wie die notwendigen Strategiepfade einer dauerhaft umweltge- \\ rechten Entwicklung im Rahmen einer europäischen Strategie bis zum Jahr \\ 2050 in konkretes Handeln umzusetzen sind.
}

$\mathrm{H}$ Von Holger Rogall ökologischen Gefahren für die natürlichen Lebensgrundlagen nicht mehr erläutert werden. Die vorliegenden Untersuchungen belegen, dass die Industriestaaten ihren Verbrauch der natürlichen Ressourcen bis 2050 um 80-90 Prozent reduzieren müssen, soll eine zukunftsfähige Entwicklung eingeleitet werden. Dieses ambitionierte Ziel wird jedoch ohne die Einführung neuer politisch-rechtlicher Instrumente mit Ausnahme der Schadstoffe in keinem Bereich erreicht werden. Die Grenzen einer nachsorgeorientierten Umweltschutzpolitik zwingen dazu, eine Ökonomie der Nachhaltigkeit einzuleiten eine Entwicklung, die zwar auf nachsorgende Techniken nicht ganz verzichtet, aber in ihrem Kern auf die ökologische Modernisierung aller Wirtschafsbereiche abzielt (1).

Die kritische Analyse des Beitrags der neoklassischen Umweltökonomie wie der nachhaltigkeitsorientierten ökologischen Ökonomie zeigt, dass die Übernutzung der natürlichen Ressourcen auf verschiedene sozial-ökonomische Faktoren des Marktversagens zurückzuführen ist (2). Sie können nicht von selbst oder lediglich über eine bessere Information der Konsumenten und Produzenten aufgehoben werden. Eine Strategie die allein auf die Konsumenten oder eine Bewusstseinsbildung setzt ist eine Illusion. Es bedarf vielmehr einer Änderung der Rahmenbedingungen, ohne die eine nachhaltige Entwicklung keine Perspektive hat. Die hierfür notwendigen politisch-rechtlichen Instrumente zur Setzung von ,ökologischen Leitplanken“ sind bereits konzipiert, bedürfen aber der konsequenteren Einführung. Als wichtigste Maßnahmen sind hierbei die neuen ökonomischen Instrumente (Ökologisierung des Finanzsystems, Naturnutzungszertifikate) sowie die ordungsrechtlichen Instrumente (Grenz- wertfestsetzungen, Höchstverbräuche und solare Baupflichten) anzusehen. Eine Akteursanalyse von den Kommunen bis zur globalen Ebene zeigt, dass die Realisierungschancen aufgrund der kurzsichtig denkenden Lobbys nicht sicher, aber bei zunehmenden globalen Problemen, Nutzung neuer Bündnispotentiale zwischen Zivilgesellschaft und Politik sowie sich bietenden Zeitfenstern durchaus gegeben sind (3).

\section{- Handlungsfelder der Nachhaltigkeit}

Über die Bedingungen einer nachhaltigen Entwicklung ist bereits viel geschrieben worden, aber an der Konkretisierung dieses Konzepts muss weiter gearbeitet werden. Sicher ist heute, dass Politik und Wirtschaft gleichermaßen gefordert sind: Die Politik muss die Rahmenbedingungen verändern, die Wirtschaft die Innovationskraft für die gezeichneten Strategiepfade aufbringen. Am Beispiel der vier Handlungsfelder Energie, Mobilität, Ressourcenschutz und Wirtschaftspolitik kann gezeigt werden, wie die ökologischen, ökonomischen und sozial-kulturellen Ziele einer nachhaltigen Entwicklung in den nächsten Jahrzehnten erreichbar sind und welche möglichen Hemmnisse dem im Wege stehen. Hierfür liegen für jedes Handlungsfeld Qualitäts- und Handlungsziele für die Jahre 2020 und 2050 vor, zum Beispiel 40 Prozent KohlendioxidReduktion bis zum Jahr 2020 und 80 Prozent bis zum Jahr 2050.

Diese sehr ambitionierten Ziele lassen sich durch einseitige Strategieansätze nicht erreichen, da sie der Herausforderung der Reduktionsziele einer nachhaltigen Entwicklung nicht gerecht werden. Nur mit der konsequenten Umsetzung der Effizienz-, Substitutions- und Suffizienzstrategie lassen sich nach den vorliegenden Szenarien die Ziele einer Ökonomie der Nachhaltigkeit bis 2050 erreichen.
Ein besonderer Schwerpunkt muss hierbei auf die wirtschaftlichen Chancen gelegt werden. Eingebettet in eine europäische Vereinigungsstrategie lassen sich aus der ökologischen Modernisierung der Volkswirtschaft erhebliche Potentiale erschließen. Zum Beispiel könnten allein im Energiesektor unter anderem durch ein umfassendes Wärmeschutzsanierungsprogramm und den massiven Ausbau der erneuerbaren Energien über eine Million Arbeitsplätze geschaffen werden.

\section{Energiepolitik}

Zwischenziele einer zukunftsfähigen Energiepolitik sind die Halbierung des Primärenergieverbrauchs bis 2050 und die Erhöhung des Anteils der erneuerbaren Energien auf mindestens 50 Prozent. Die vorliegenden Studien belegen, dass diese Ziele durch den konsequenten Ersatz konventioneller Geräte und Anlagen durch effiziente Techniken zu erreichen sind. Die Betrachtung der technischen und wirtschaftlichen Potentiale zeigt, welche großen Beiträge effiziente Kraftwerke, insbesondere GuD-Anlagen und Blockheizkraftwerke, hierzu leisten können. Sie kommen auf Wirkungsgrade von 90 bis 95 Prozent gegenüber 35 bis 42 Prozent konventioneller Kondensationskraftwerken. Da in den nächsten 20 Jahren die Mehrzahl der europäischen Kraftwerke erneuert werden muss, könnten erhebliche Effizienzgewinne realisiert werden. Umso unverständlicher sind die schwachen Minderungsziele des gerade verabschiedeten Kohlendioxid-Emissionhandelssystems. Das System kann daher nur als Probelauf akzeptiert werden.

Ein weiteres Beispiel für die großen wirtschaftlichen und umweltpolitischen Chancen einer ökologischen Modernisierung der Volkswirtschaft sind die enormen Beschäftigungschancen und Kohlendioxid-Minderungspotentiale wenn der europäische Gebäudebestand systematisch wärmeschutzsaniert wird, das Energieeinsparpotential liegt hier bei 70 bis 77 Prozent.

Der nächste große Sektor betrifft den Ausbau der erneuerbaren Energieträger, die langfristig - die Ausschöpfung der Effizienzpotentiale vorausgesetzt - die gesamte Energieversorgung der Erde übernehmen könnten. In Deutschland sind bis zum Jahr 2050 selbst unter erhöhten Naturschutzstandards insbesondere durch die Nutzung der Sonnenenergie, Windkraft und Biomasse circa 50 Prozent der Energieversorgung technisch und wirtschaftlich darstellbar. 


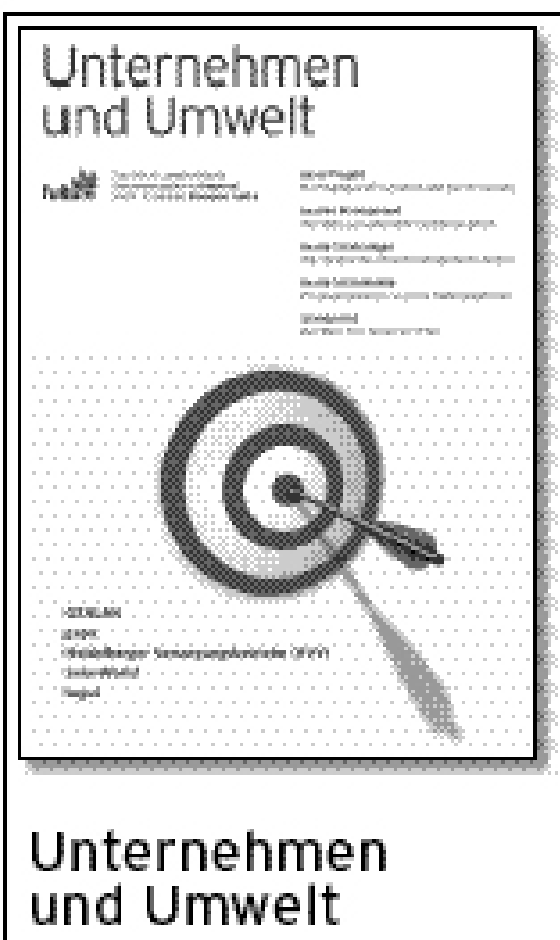

... ist die Zeitsctrift für umweltorientierte Unternehmerspolitik von future e.V.

... bietet Fachinformationen zum Unrweltsctutz und Best-Practice-Beispiek aus Unternehmen.

... bietet viermal im lahr Urternetrmensbeipiek, Literatur- und Verantaltungshinweis.

Mit enem Schwerpunktthema in jedem Heft. Zum Beispid:

\section{3-4/04: Nachindtiges Wirtschatten and Benchmarking \\ 2/04: Envissionshundel \\ 1/04: Bildung fiv Nachhaltige Entwickhng \\ 4/03: Konpeterm mochhaitig entwickein \\ 3/03: Nactihaltigke it and Regrion \\ 2103: Kapital fir Nochthaltigherit \\ 1/03: Energieeffrzienz \\ 3-4//O2: Benchurarking for Sustainability \\ 2102: Ohomurheting \\ 1/02: Nacthaltigkeit und Gewerbebsu}

Bestellen Sie ihr kostenloses

Probe-Abo im Internet unter

www.fut ure-ev. de

fukure e.V. Büro Bochum

Avm Varerhalt 123

44797 Bochum

Fon: 0234/9799513

Fax: 0234/9799514

bochumefuture-ev.de

www. future-ev.de

\section{Mobilität und Ressourcenschutz}

Auch die Untersuchung der Mobilität zeigt, dass aufgrund der hohen sozialen Kosten, die der Verkehr heute mit sich bringt, eine Trendumkehr unverzichtbar ist. Wie in der Energiepolitik sind auch hier große Effizienzsteigerungs-, Substitutions- und Suffizienzpotenziale vorhanden. Die Einführung der hierfür notwendigen Instrumente setzt allerdings erhebliche Anstrengungen der politischen und gesellschaftlichen Akteure voraus.

$\mathrm{Zu}$ dem gleichen Resultat kommt die Analyse des Handlungsfeldes Ressourcenschonung inklusive EcoDesign und Abfallpolitik. Auch wenn sich dieser Bereich als deutlich komplexer erweist als zum Beispiel die Energiepolitik, sprechen die umwelt- und wirtschaftsrelevanten Potenziale einer Ressourcenschonungspolitik doch für ein verstärktes Engagement. Heute betragen nach den Berechnungen des Wuppertal Instituts die Kosten für die Nutzung der natürlichen Ressourcen in Deutschland etwa 750 Milliarden Euro pro Jahr. Ein großer Teil dieser Mittel könnte durch eine Politik der ökologischen Modernisierung dauerhaft vermieden werden und somit für andere Bereiche zur Verfügung stehen.

\section{- Wirtschaftspolitik}

Die traditionellen Wirtschaftsschulen wie Neoliberalismus und Keynesianismus verfügen über keinerlei Lösungsansätze für die globale Zerstörung der natürlichen Lebensgrundlagen und verschärfen das Problem der Armut (Neoliberalismus) oder der Staatsverschuldung (Keynesianismus). Auch wenn eine ausformulierte Schule einer Ökonomie der Nachhaltigkeit noch nicht existiert, gibt es doch mehrere Strategieansätze, die einen deutlichen Beitrag zur Erreichung des Zieldreiecks der Nachhaltigkeit leisten könnten. Auf das Ziel einer Vollbeschäftigung sollte auf absehbare Zeit nicht verzichtet werden, allerdings nicht in Form der heutigen Vollerwerbstätigkeit. Die Gefahr, dass die Modelle der Eigenarbeit mit Bürgergeld in Wirklichkeit nur zu einer Sozialhilfe mit anderem Namen führen und eine Gesellschaft von „Brot und Spielen“ zur Folge haben, ist einfach zu groß. Auch für dieses Handlungsfeld zielen einzelne Strategieansätze zu kurz. Vielmehr müssen mehrere Wege zugleich beschritten werden: erstens ökologische Modernisierung der Volkswirtschaft, zweitens kostenneutrale Verteilung der Arbeit, insbesondere Teilzeitarbeit nach dem Niederländischen Modell; drittens Finanzierung von Arbeit statt Arbeitslosigkeit, unter anderem Ausbau des Bildungssektors durch Subventionsab- bau, gemeinnützige Arbeit, nach dem Skandinavischen Modell; viertens internationale sozialökonomische Mindeststandards.

\section{Fazit}

Ob das bisherige Marktversagen in absehbarer Zeit durch politisch-rechtliche Instrumente ausreichend vermindert werden kann und die dargestellten Strategiepfade tatsächlich beschritten werden, kann nicht voraus gesagt werden. Im Zuge der absehbaren Verschärfung der globalen Umweltkrise wird dies aber für möglich gehalten. Am Beispiel der Ökologisierung des Finanzsystems könnte so eine „fünffache Dividende“ einer Ökonomie der Nachhaltigkeit realisiert werden: weniger Umweltbelastung durch Senkung des Ressourcenverbrauchs; mehr Arbeitsplätze durch die Senkung der Sozialabgaben sowie den Anreiz, ressourceneffizientere Produkte zu entwickeln und die Gebäude zu isolieren; Stärkung der sozialen Sicherungssysteme durch Verbreiterung der Finanzierungsbasis und Abbau der Arbeitslosigkeit; Verringerung der wirtschaftlichen Abhängigkeit von Ressourcenimporten und hierdurch auch ein Beitrag für die internationale Friedenssicherung. Der Verzicht auf die weitere Erhöhung der Öko-Steuern wird daher nur kurzfristig - zum Beispiel bis zum Wahlabend 2006 - akzeptiert, ansonsten wäre das Rot-Grüne-Projekt wohl gescheitert. Eine derartige Prognose ist aber viel zu verfrüht.

\section{Anmerkungen}

(1) Vgl. Rogall, H.: Ökonomie der Nachhaltigkeit - Handlungsfelder für Politik und Wirtschaft. Wiesbaden 2004.

(2) Rogall, H.: Neue Umweltökonomie - Ökologische Ökonomie. Opladen2002

(3) Rogall, H): Akteure der nachhaltigen Entwicklung, München 2003.

\section{Der Autor}

Dr. Holger Rogall (MdA) ist Professor für Umweltökonomie an der FHW Berlin und Vorsitzender der Gesellschaft für Nachhaltigkeit (GFN).

Kontakt: Zehntwerderweg 124 a, 13469 Berlin. Tel. 030-40213 56, E-Mail: H.Rogall@t-online.de 
(c) 20I0 Authors; licensee IÖW and oekom verlag. This is an article distributed under the terms of the Creative Commons Attribution Non-Commercial No Derivates License (http://creativecommons.org/licenses/by-nc-nd/3.o/), which permits unrestricted use, distribution, and reproduction in any medium, provided the original work is properly cited. 